\title{
Clinical and Pathological Features of C3 Glomerulonephritis
}

\author{
Qi Jiang, Yingying Liu* \\ Department of Nephrology, China-Japan Union Hospital of Jilin University, Changchun, Jilin, 130033, China \\ ${ }^{*}$ Corresponding author
}

Keywords: C3 glomerulonephritis, Clinical manifestation, Case features, Clinical value

\begin{abstract}
The clinical manifestations and pathological features of C3 glomerulonephritis were analyzed in order to provide some references for the clinical diagnosis and treatment of C3 glomerulonephritis. The paper selected 60 cases of C3 glomerulonephritis from January, 2015 to December, 2017 in China-Japan Union Hospital of Jilin University selected for clinical study. According to the clinical data, the clinical manifestations and case characteristics of the patients were statistically analyzed. In a word. there were 20 cases with the first symptom of hematuria, 40 cases with hematuria and proteinuria as the first symptom, 30 cases of nephritic syndrome, 10 cases of nephrotic syndrome and 60 cases of child complement C3 decreased and the complement C4 was normal. The positive deposition of complement C3 was observed in 60 children with immunofluorescence; there were 50 cases of Mesangial proliferative glomerulonephritis and 10 cases of endocapillary proliferative glomerulonephritis under light microscope; electron dense deposit was found in 30 cases under electron microscope, and no electron dense deposit was found in 30 cases. The routine treatment of nephritis was given, and 2 crescent forming patients were treated with glucocorticoid. All patients with C 3 glomerulonephritis were followed up for 6 to 42 months and the prognosis was good. To sum up, in children with C3 glomerulonephritis, hematuria and (or) proteinuria was the main manifestation. Immunofluorescence was found in C3 strongly positive deposition. The light microscopy showed that mesangial proliferative glomerulonephritis was dominant, and electron microscopy showed electron dense deposit and short-term prognosis.
\end{abstract}

\section{Introduction}

C3 glomerulonephritis is a newly recognized disease, and its clinical manifestation lacks characteristic. The immunopathology was regarding c3 deposition as main body and electron microscopy was used to observe the deposition of electron dense matter in glomerular capillary loop. At present, there is no effective treatment, and its pathogenesis needs to be further studied. In recent years, some experts have clearly proposed that C3 glomerulopathy can be used as a general term for a class of diseases, including compact deposition, C3 glomerulonephritis, familial type III membranous proliferative glomerulonephritis and CFHR5 nephropathy.

\section{Materials and Methods}

\subsection{Clinical Data}

From January, 2015 to December, 2017, 60 cases of C3 glomerulonephritis were selected for clinical study, including 38 male patients and 22 female patients. The average age was $32.6 \pm 5.4$ years. Exclude all patients with severe cardiovascular disease, oncology, blood disease, immune disease, and mental disorders, and ensure that the results of the study are not affected.

\subsection{Methods}

(1) Clinical observation indicator: Clinical observation indicator included preinfection, initial symptoms, concomitant symptoms and urine routine, 24h-urinary protein quantification, blood complement, hemolytic streptococcin $\mathrm{O}$, renal function and other laboratory indexes (all the laboratory results were the first laboratory results of the children after admission). (2) Criteria for determination of clinical indicator. Hematuria: Microscopic hematuria is expressed as urinary sediment count $(\mathrm{RBC}) \geq 5 / \mathrm{HP}$, and naked hematuria is expressed as RBC $>50$ percent HP. 
Proteinuria: If the $24 \mathrm{~h}$-urinary protein is showing $\geq 0.2 \mathrm{~g}$, and at the same time hypoalbuminemia is showing $\leq 25 \mathrm{~g} / \mathrm{L}$, it can be diagnosed as nephrotic syndrome. Hypocomplement: It refers to the blood complement $\mathrm{C} 3 \leq 0.75 \mathrm{~g} / \mathrm{L}$. Antistreptococcal hemolysin O (ASO) was positive: $>200$ $\mathrm{U} / \mathrm{mL}$; renal dysfunction: Serum creatinine (SCr) was based on the level of creatinine in healthy children with or without usea nitrogen (BUN) $(\geq 7 \mathrm{mmol} / \mathrm{L})$. Hypertension: Children of school age $\geq 130 / 90 \mathrm{mmHg}(1 \mathrm{mmHg}=0.133 \mathrm{kPa}$, and preschool children $\geq 120 / 80 \mathrm{~mm} \mathrm{Hg}$. (3) Renal biopsy. If the children has a long medical history or is still in hematuria and or proteinuria for 2 weeks after admission, the sonographic guidance is used to perform renal puncture after obtaining the informed consent of the family. (4) Pathological examination. Pathological biopsy of renal tissue was sent to the pathology room of urological diagnosis and treatment center of the first Hospital of Jilin University. The specimens were embedded in paraffin, sectioned, stained with hematoxylin and eosin (HE), and stained with Schiff sulfonic acid (PAS) staining with Hexamamine periodate (PASM) and Masson trichrome; while, the renal tissues were stained with IgG, IgA, IgM, C3, C1q and Fibrin. The intensity and location of immunoglobulin and complement deposition were observed. The results of immunofluorescence examination were classified as $-\sim++++$ according to fluorescence intensity grade under fluorescence microscope. Electron microscope was sent to the Department of Pathology, Peking University Department of Medicine to observe the features of glomeruli, renal tubules, interstitial lesions and electron dense matter deposition.

\section{Results}

\subsection{Clinical and Laboratory Findings of All Patients with C3 Glomerulonephritis}

Hematuria as the first symptom was 20 cases and hematuria and proteinuria as the first symptom was 40 cases. There were 30 cases of nephritis syndrome and 60 cases of nephrotic syndrome with decreased complement C3 and normal complement C4.

\subsection{Statistical Analysis of Pathological Features in all Patients with C3 Glomerulonephritis}

The positive deposition of complement C3 was observed in 60 children with immunofluorescence; there were 50 cases of Mesangial proliferative glomerulonephritis and 10 cases of endocapillary proliferative glomerulonephritis under light microscope; electron dense deposit was observed in 30 cases under electron microscope and no electron dense deposit was found in 30 cases; treatment and prognosis; two patients with crescent formation were treated with glucocorticoid. All patients with C3 glomerulonephritis were followed up for 6 to 42 months and the prognosis was good.

\section{Discussions}

In recent years, with the improvement of living standard, the incidence of C3 glomerulonephritis increased significantly, which seriously affected the health and quality of life of patients. At present, many domestic scholars have begun to study C3 glomerulonephritis, whch mainly discusses its clinical manifestations and case characteristics, so that to provide a theoretical reference for the clinical diagnosis and treatment of C3 glomerulonephritis.

In 1990s, MPGN with clear underlying diseases such as cold globulinemia, paraglobulinemia, autoimmune disease and infection was defined as secondary MPGN. The other so-called primary MPGN are divided into 3 types according to the location of the electron density deposited in the glomeruli. First, Type I: Electron dense materials were deposited in glomerular Mesangial area and GBM. The typical immunopathologic changes were immunoglobulin with complement deposition. Second, Type II: Electron dense materials were deposited in GBM and (or) glomerular Mesangial area. The typical immunopathologic changes were C3 deposition. Third, Type III: It can be divided into Burkholder subtype and Strife-Anders subtype. The electron density of the former was deposited subcutaneously and epithelially in GBM of glomerular Mesangial area, and the typical immunopathologic changes were immunoglobulin with complement deposition. The electron 
densification of the latter can penetrate the whole layer of GBM, and the typical immunopathologic changes are C3 with or without immunoglobulin deposition. It was later found that some type II MPGN did not appear as MPGN under light microscope, so it was defined as dense deposit disease (DDD). It is more reasonable than the definition of type II MPGN. In addition, some type I and III MPGN can only be deposited by C3, which poses a challenge to traditional typing. A breakthrough was the discovery of AP complement components, gene mutations or defects in complement regulatory proteins in patients with single C3 deposition. These diseases are mediated by the persistent abnormal activation of AP, and it is different from the traditional type I and III MPGNs, which are mediated by immune complexes.

During the period of improving the therapeutic effect of C3 glomerulopathy, the pathogenesis of C3 glomerulonephritis has been studied. Its pathogenesis and pathological features are different from those of common glomerulonephritis. It is believed that C3 glomerulonephritis is a proliferative glomerulonephritis caused by the continuous activation of complement $\mathrm{C} 3$ due to abnormal complement bypass pathway. Complement C3 was deposited in the glomerulus and the level of serum complement C3 decreased, and the complement C4 usually did not change significantly. Some of the children with C3 glomerulonephritis were associated with elevated ASO, which should be distinguished from glomerulonephritis after acute streptococcal infection. Foreign studies suggest that children with acute streptococcal glomerulonephritis may exhibit severe renal dysfunction and lower C3 levels in the early stages of the disease. C3 glomerulonephritis is a mild and progressive disease. It has also been suggested that streptococcal infection may trigger $\mathrm{C} 3$ activation to cause or accelerate disease progression. At present, the pathogenesis of this disease is not clear. The research focus at home and abroad is the genetic background and gene mutation of various complement factors and regulatory proteins. For example, familial C3GN and complement $\mathrm{H}$ factor associated protein 5 (CFHR5) nephropathy with gene change of factor $\mathrm{B}$, and so on, have different conclusions. C3 glomerulonephritis mainly includes DDD and C3GN. DDD was characterized by the uniform deposition of the dense layer of glomerular basement membrane like the strong osmiophilic ribbons under electron microscope. Other C3 glomerulopathy except DDD is classified as C3GN.

By reviewing domestic and foreign C3 glomerulonephritis related research progress combined with the diagnosis and treatment of patients in our hospital after studying statistical data, it showed that the clinical and laboratory findings of 60 patients with C3 glomerulonephritis were as follows: Hematuria was the first symptom in 20 cases and hematuria and proteinuria as the first symptom in 40 cases, among which there are 30 cases of nephritis syndrome, 10 cases of nephrotic syndrome and 60 cases of children with decreased complement C3; complement C4 was normal. The pathological features are as follows: The positive deposition of complement C3 was observed in 60 children with immunofluorescence. There were 50 cases of mesangial proliferative glomerulonephritis and 10 cases of endocapillary proliferative glomerulonephritis under light microscope; electron dense deposition was observed in 30 cases under electron microscope and no electron dense deposition was found in 30 cases; treatment and prognosis; two patients with crescent formation were treated with glucocorticoid. All patients with $\mathrm{C} 3$ glomerulonephritis were followed up for 6 to 42 months and the prognosis was good. The results showed that the main manifestations of C3 glomerulonephritis were hematuria and (or) proteinuria. Immunofluorescence showed strong C 3 positive deposition, and mesangial proliferative glomerulonephritis was predominant in light microscopy. Electron microscope can show electron dense material deposition, and short term prognosis is better. This is close to the research conclusions of experts and scholars at home and abroad, and has a certain degree of clinical reference value.

At present, the diagnosis and treatment of C3 glomerulonephritis is mainly limited to case studies and retrospective studies. This study is also a retrospective study of the clinical data of patients in our hospital. There is a lack of randomized controlled study. At the same time, the number of sample cases in this study is small, which needs to be further studied. Therefore, it is expected to provide a more effective reference for clinical. 


\section{References}

[1] Zhang Haitao, Chen Huiping, Zeng Caihong, Deng Kangping, Li Shijun, Zheng Chunxia, Liu Zhihong. Clinical and Pathological Features of C3 Glomerulonephritis [J]. The Journal of Kidney Disease and Dialysis Kidney Transplantation, 2011, 20(04): 307-311+337.

[2] Wang Jinquan. Heterogeneity of Clinical Manifestations and Pathological Changes in C3 Glomerulonephritis [J]. The People's Liberation Army Medical Journal, 2014, 39(12): 998-1003.

[3] Ren Guoqing. Clinical and Pathological Features of C3 Glomerulonephritis [J]. China Health Standardization: 2015: 60.80: 50-51.

[4] Wang Lili, Zhao Kaishu, Li Qingmei, Ma Qingshan. Clinical and Pathological Analysis of 6 Cases of C3 Glomerulonephritis in Children [J]. Chinese Journal of Practical Pediatrics, 2017, 32(05): 374-378.

[5] Yang Fan, Deng Li, Chen Zelin, Wan Ziqing, Rong Yi, Mo Ying, Jiang Xiaoyun. C3 Glomerulonephritis in Children and its Literature Review [J]. Chinese Journal of Clinical Medicine for Women and Children (Electronic Edition), 2017, 139050: 574-579. 